\title{
Statistical learning reflects inferences about unique predictive relations
}

\author{
Anna Leshinskaya ${ }^{1},{ }^{2}$, and Sharon L. Thompson-Schill ${ }^{1}$ \\ ${ }^{1}$ Psychology Department, University of Pennsylvania \\ ${ }^{2}$ University of California, Davis
}

\begin{abstract}
The mind adeptly registers statistical regularities in experience, often incidentally and implicitly. We used a visual statistical learning paradigm to study what kinds of statistics it spontaneously computes in such conditions. We found that participants' learning of pairwise predictive relations was best explained by an inferentially sophisticated quantity, $\Delta P$, which reflects whether a high conditional probability between an event pair is unique. We showed that uniqueness can be reduced by either a strong competing predictor or an overall high base rate of the outcome. Both can result from normalization: if predictors of the same effect trade off, a predictor must raise the probability of the effect more than the others to be effective. Adding normalization to the Rescorla-Wagner learning model captures these results. We argue that the uniqueness of a relation is an intrinsically important statistical property that governs learning without incentive or deliberation.
\end{abstract}

Keywords: statistical learning; associative learning; causal reasoning; Rescorla-Wagner model; backward blocking

\section{INTRODUCTION}

Our minds are naturally adept at registering patterns in experience. For example, we spontaneously encode predictive relations among sequentially presented stimuli (Saffran, Aslin, \& Newport, 1996; Turk-Browne, Jungé, \& Scholl, 2005). This phenomenon, statistical learning, takes place without conscious effort, feedback, or reward. It suggests the existence of a learning mechanism operating in the background to produce mental models of our world and offers us the opportunity to ask what kinds of models it is intrinsically infers. Here, we test the possibility that incidental statistical learning is best characterized as an attempt to identify unique predictive relations, rather than simply strong ones.

The literatures on more deliberative or incentivized forms of learning, such as causal reasoning, contingency learning, and classical conditioning (for a review: Mitchell, De Houwer, \& Lovibond, 2009) describe a core phenomenon of some inferential complexity: learners do more than register that two stimuli co-occur, but also compute whether they predict each other uniquely and independently, as if attempting to determine a causal model. Suppose two events A and B coincide, such that after most occurrences of A, B occurs. However, B

*Address correspondence to: Anna Leshinskaya Department of Psychology University of Pennsylvania 425 S. University Ave, Stephen A. Levin Bldg. Philadelphia, PA, 19104 anna.leshinskaya@gmail.com also occurs without $\mathrm{A}$ at a very high rate. One would not represent a strong link between $\mathrm{A}$ and $\mathrm{B}$ in this case. This consideration is captured by a foundational learning formula, $\Delta P$ (Allan, 1980; Rescorla \& Wagner, 1972; Shanks, 1985):

$\Delta P=P(B \mid A)-P(B \mid \sim A)$

This equation states that learning is a product of both how often B follows A, as well as how often B appears without it. This consideration is at the core of the Rescorla-Wagner learning rule, which accounts for several phenomena concerning uniqueness in classical conditioning and contingency learning, termed cue selection or blocking (Kamin, 1968; Rescorla \& Wagner, 1972; Shanks, 1985). For example, in forward blocking, after learning that stimulus B coincides with an outcome (denoted $\mathrm{B}+$ ), learning that both $\mathrm{A}$ and $\mathrm{B}$ yield this outcome $(\mathrm{AB}+)$ leads to weak associative strength between the outcome and $\mathrm{A}$; this is less the case than if participants saw only $\mathrm{AB}+$ trials. Thus, judgments about the predictive importance of $\mathrm{A}$ are affected by whether a different stimulus is also predictive. At a broad level of description, this implies that learners care about the unique predictiveness of stimuli, a more complex statistic than conditional probability.

Surprisingly, it is not known whether this uniqueness principle governs learning in more incidental learning 
contexts like statistical learning tasks, although it has been reported in a paradigm somewhere in between statistical learning and conditioning (Sobel \& Kirkham, $2006,2007)$. Prior work in statistical learning has shown that learners opt to track the conditional probability that B follows A (transition probability), rather than the joint probability (total number of A-B pairs) when these are both available (Fiser \& Aslin, 2002). However, whether such learning might instead be best described as being sensitive to $\Delta P$ has not been tested nor considered in subsequent work (Buchsbaum, Griffiths, Plunkett, Gopnik, \& Baldwin, 2015; Endress \& Langus, 2017; Frank, Goldwater, Griffiths, \& Tenenbaum, 2010; Orbán, Fiser, Aslin, \& Lengyel, 2008).

Whether statistical learning is governed by similar principles as classical conditioning and causal reasoning is not a trivial question because statistical learning paradigms differ substantially from these others. Causal reasoning tasks direct participants to look for evidence of causality and the evidence they use to do so is fully encoded by them (Cheng, 1997; Griffiths \& Tenenbaum, 2005). These tasks thus reflect reasoning, rather than learning. In other contingency learning tasks, including classical conditioning, the expectation of a reward is also governed by $\Delta P$, but it is also possible that such expectations might arise from a deliberative, reasoned process over explicitly available information (Mitchell et al., 2009; Mitchell, Lovibond, Minard, \& Lavis, 2006). The fact that stimuli in such tasks (shocks or rewards) are inherently value-laden strongly incentivizes attention to the learning task and to the relevant outcome to be predicted; the computation of such predictors is thus very likely intentional and the product of reasoning. By contrast, in statistical learning paradigms, participants passively observe streams of events, with no strategic advantage or instruction to identify predictive relations of any sort. The contents of learning are also typically unavailable for verbalizable report (Brady \& Oliva, 2008; Kim, Seitz, Feenstra, \& Shams, 2009), likely because the number of stimuli, and their rapid, continuous presentation, prevents explicit tracking of their rates of co-occurrences; and because there is no prior indication of what is a 'cue' and what is an 'outcome'. It is not a given that the principles guiding explicit judgment about the strength of a relationship are the same as those that guide the formation of the memory of that relationship. It thus remains an open question whether in incidental learning situations, learners use $\Delta P$ rather than a simpler statistic like conditional probability, when both are available in the input.

Another unique feature of statistical learning tasks is that stimuli often appear one by one, rather than appearing in compounds like in traditional blocking paradigms (i.e., where learning $\mathrm{AB}+$ blocks the learning of $\mathrm{A}+$ ). In sequentially presented statistical learning trials, the uniqueness of a prediction from A to the outcome would thus depend on whether the outcome follows stimuli other than A (e.g., B+) on separate, individual occasions. Although this still captures the deeper principle behind blocking (which we term uniqueness), it comes about in a quite different form, one which has not been previously tested. Indeed, as we detail below (in the section titled Computational Modeling), the influence of separate $\mathrm{B}+$ occurrences on $\mathrm{A}+$ representations requires a more sophisticated computation than the standard Rescorla-Wagner model. Thus, it is possible for all of the above reasons that learning taking place under the conditions of statistical learning is not, in fact, governed by the uniqueness principle.

Our experiments demonstrate that it is. In Experiment 1, we show that participants fail to use conditional or joint probabilities to learn about the relation between two events (which we call a cause and an effect) when the $\Delta P$ between them is low. Experiments $2 \& 3$ demonstrate that non-uniqueness can arise from either a strong alternate predictor, or an overall high base-rate of the effect. We then adapt the Rescorla-Wagner learning model (Rescorla \& Wagner, 1972) to account for these results. Overall, we suggest that during incidental learning, conditional probabilities between events A and $\mathrm{B}$ are spontaneously normalized by the overall rate of occurrence of event B otherwise, and that learning takes place when these relative values are high. Thus, statistical learning should be best described as governed by $\Delta P$, not conditional probabilities as largely assumed in prior work, suggesting that the uniqueness of a relation may be an inherently important statistical quantity that the mind naturally computes as part of the way it forms memories, even without deliberative reasoning.

\section{EXPERIMENT 1}

We tested whether learning in a visual statistical learning (VSL) task is affected by uniqueness. Participants saw two distinct event sequences, each composed of a non-overlapping set of animated events (Figure 1A). Each sequence contained one strongly predictive event pair - which we call a cause and an effect - such that cause was almost always ( $\sim 98 \%$ of the time) followed by the effect (visually summarized in Figure 1D, and described in detail in Table 1). Thus, for the cause-effect event pairs in both sequences, the first term in the $\Delta P$ formula, $\mathrm{P}$ (effect|cause), was equally high . However, the sequences differed in the value of the second term, $\mathrm{P}$ (effect $\mid \sim$ cause $)$. In the high $\Delta P$ sequence, this term was low, making $\Delta P$ high. In the low $\Delta P$ sequence, this term was high, making $\Delta P$ low. This was done by allowing the effect to follow other events in the sequence more often, lowering its uniqueness. Thus, in the two conditions, the relationship between cause and effect was matched in terms of their conditional probability and their chunk frequency: the number of times a cause-effect 
pair appeared. What varied was how uniquely the cause, rather than other events, predicted the effect.

\subsection{Methods}

\subsubsection{Participants}

100 participants were recruited and tested via Amazon Mechanical Turk. Participants provided electronic consent and procedures were approved by the Institutional Review Board of the University of Pennsylvania. Compensation was $\$ 3$, with a bonus of up to $\$ 2.50$ based on cover task accuracy. Participants were excluded if they had previously participated in a related experiment or this one (10), for failing an attention measure (8), for missing data (1) or reporting a technical glitch (1). Data from the remaining 80 participants are reported here (43 female, age $M=33$, range $19-62$ ).

\subsubsection{Stimuli \& Design}

Stimuli were sequences of animated events (1.2 s GIFs; Figure 1) which took place surrounding or involving a continually present object. Each participant viewed both sequence conditions, low $\Delta P$ and high $\Delta P$, each involving a distinct object and containing a distinct set of 4 different event types. Each event type had a common version (Figure 1B, top row) and a slightly visually distinct rare alternate (Figure 1B, bottom row), for the purposes of the cover task to keep participants engaged (described below). Sequences were specified over the event types, with rare alternates randomly replacing their regular version $10 \%$ of the time. A static event (object standing still for $3.6 \mathrm{~s}$ ) also appeared; this provided short breaks in the sequence.

The specific event types and object shape assigned to each sequence condition and role (as Cause, Effect, or Random) were selected randomly for 20 sets of materials; then, yoked materials were created by swapping these assignments between the two sequence types. There was one cause, one effect, and two random events. Each yoked set was then used twice, once for each possible order of presentation of the two sequences, creating materials for 80 participants fully counterbalanced for order and stimuli.

Events were concatenated into 500-event-long sequences for each condition, which was then split into a passive preview (100) and two cover task segments (200 each). The order of events in the sequence was governed by a pairwise transition matrix, one for each condition (Table 1). The matrices specified the conditional probability of showing any particular event type on trial n, given the event type shown on trial $n$ - 1 . These matrices also determined the base rate (overall frequency or probability of occurrence) of each event, shown below the transition matrices in Table 1. Both matrices specified that the effect followed the cause with a high conditional probability $(\sim 98 \%)$. The critical manipulation was on the probability of the effect given events other than the cause, $P$ (effect $\mid \sim$ cause), seen in the columns for the effect event. This was computed as the probability of the effect appearing on trial $n+1$, given that any non-cause event appeared on trial $\mathrm{n}$. Accordingly, $P$ (effect $\mid \sim$ cause $)$ was subtracted from $P$ (effect|cause) to yield $\Delta P$ for the cause-effect relationship; this was higher in the high $\Delta P$ sequence $(97 \%)$ than in the low $\Delta P$ sequence $(61 \%)$.

Sequences for each of the 40 unique subject materials were generated stochastically according to idealized transition matrices, which are very similar to the mean obtained matrices shown in Table ??. The obtained sequences in the two conditions were closely matched on the conditional probability of seeing the effect given the cause (high, $97.8 \%$; low, 97.4\%), and their chunk frequency (number of times participants saw a cause-effect pair; high, 63.28; low, 62.18). The conditional probability of the cause given the effect was lower for the low $\Delta P$ sequence (high, .074, low, .027) so that the number of times they saw the effect-cause pair $(h i g h, 5.12 ;$ low, 6.02$)$ was relatively matched. We address any concerns about minor differences in the sequence properties in the Results.

\subsubsection{Procedure}

Cover task. Participants' cover task was to learn to identify the 'common' vs. 'rare' version of each event type (Figure 1). To ensure comprehension of what is an 'event', each sequence condition began with a display showing images of both versions of each event type (but not which were which). This was followed by a 20-event practice sequence requiring them to hit the spacebar in response to the start of each distinct event; $80 \%$ accuracy was required to continue. Subsequently, to learn which version was common vs. rare, they watched a 100-event 'preview' sequence segment where no response was required. This was followed by the two 200-event task segments in which they were asked to hit ' $\mathrm{r}$ ' for rare and 'o' for common. Feedback was given on cover task accuracy. The same procedure was repeated for the other sequence condition: static images of the new events, practice, preview, and cover task segments. The order of the sequence types was counterbalanced across subjects within the yoked stimulus set.

Forced-choice test. Following all sequence presentations, a surprise forced-choice test probed participants' knowledge of the cause-effect relations in the two conditions, using the common event versions only (Figure 1C). Questions were blocked by condition, with order of blocks matching the order of presentation during the task (and thus also perfectly counterbalanced across participants). The critical questions showed the cause followed by the effect on one side, and the effect followed by the cause on the other; participants had to choose the event pair that seemed more typical or familiar. This question was repeated 3 times for each condition; accuracy was aver- 
A

Exposure task: "Decide if the event is common or rare"

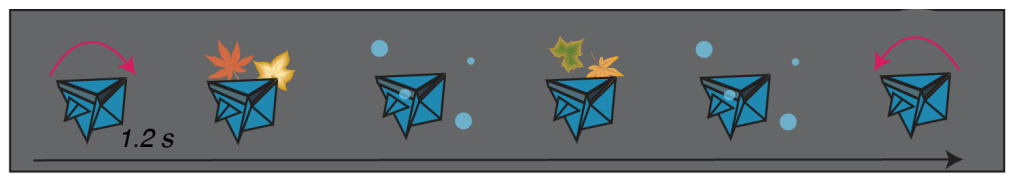

500 events / 10 minutes over 3 segments

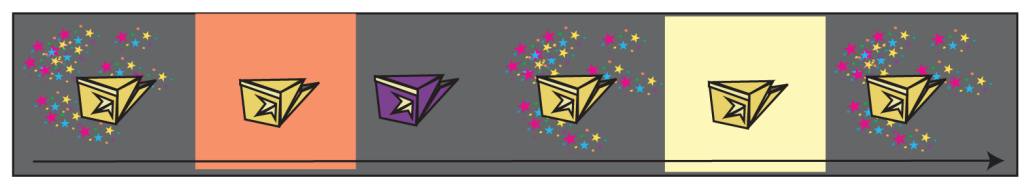

B common alternates
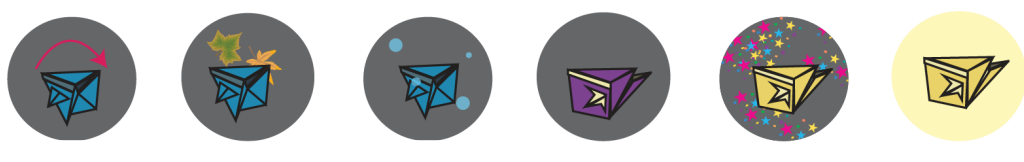

rare alternates
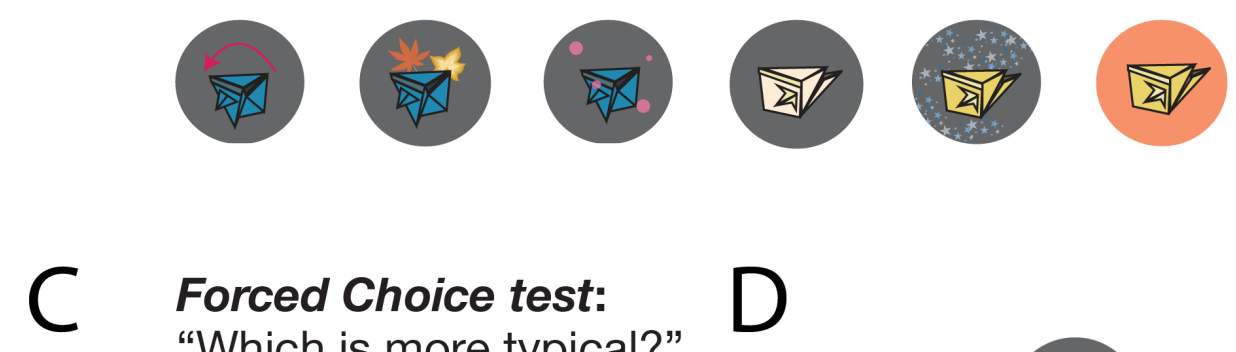

Forced Choice test:

"Which is more typical?"

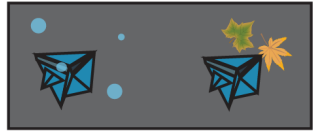

cause-effect

VS.

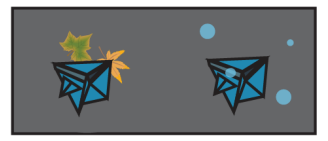

D

effect-cause

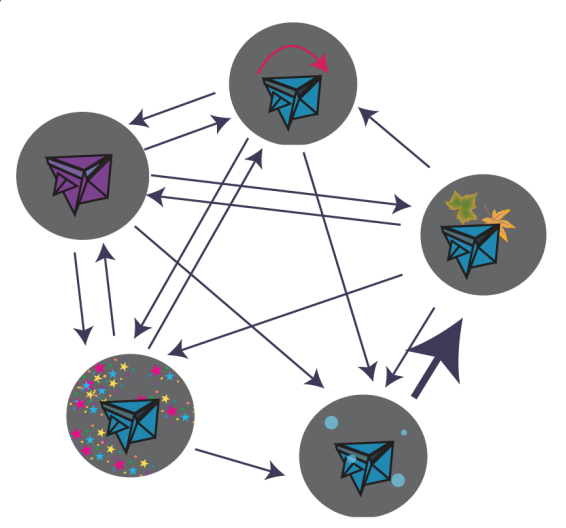

Figure 1. A. Example of cover task, in which participants viewed two distinct, 500-event-long sequences of animated events and decided if they saw a common or visually altered version of an event. B. Example stimuli with the common and rare visual alternates. C. Learning was assessed with a forced-choice test (over common events), comparing highly probable vs. unlikely transitions. D. Visual depiction of transition probabilities (which events are likely to follow each other), in which a 'cause' event (here, bubbles) strongly predicts another, 'effect' event (here, leaves). Transitions are fully independent of appearance of rare vs. common visual alternates. 
Table 1 Transition/conditional probabilities between all pairs of events and overall frequencies of each event in the two sequence conditions in Experiment 1, High $\Delta P$ and Low $\Delta P$. In the transition matrices, rows show the event type at trial n; columns show the probability of showing each event type at trial $n+1$. All values shown describe the actual materials shown to participants and closely resemble the idealized values used to construct the materials.

High $\Delta P$ Condition $(\Delta P=97 \%)$

\begin{tabular}{|c|c|c|c|c|c|}
\hline \multicolumn{6}{|c|}{ Probability at Trial $n+1$} \\
\hline Event Type at Trial $n$ & Cause & Effect & Random 1 & Random 2 & Static \\
\hline Cause & 0.00 & 0.98 & 0.00 & 0.00 & 0.02 \\
\hline Effect & 0.07 & 0.00 & 0.47 & 0.45 & 0.00 \\
\hline Random 1 & 0.15 & 0.01 & 0.38 & 0.41 & 0.05 \\
\hline Random 2 & 0.16 & 0.01 & 0.40 & 0.38 & 0.05 \\
\hline Static & 0.28 & 0.01 & 0.28 & 0.29 & 0.14 \\
\hline \multicolumn{6}{|l|}{$P($ effect $\mid \sim$ cause $)=.01$} \\
\hline \multicolumn{6}{|c|}{ Overall Event Frequency (Base Rate) } \\
\hline $\begin{array}{l}\text { Cause } \\
0.13\end{array}$ & $\begin{array}{l}\text { Effect } \\
0.13\end{array}$ & $\begin{array}{l}\text { Random } 1 \\
0.35\end{array}$ & $\begin{array}{l}\text { Random } 2 \\
0.35\end{array}$ & $\begin{array}{l}\text { Static } \\
0.04\end{array}$ & \\
\hline
\end{tabular}

Low $\Delta P$ Condition $(\Delta P=61 \%)$

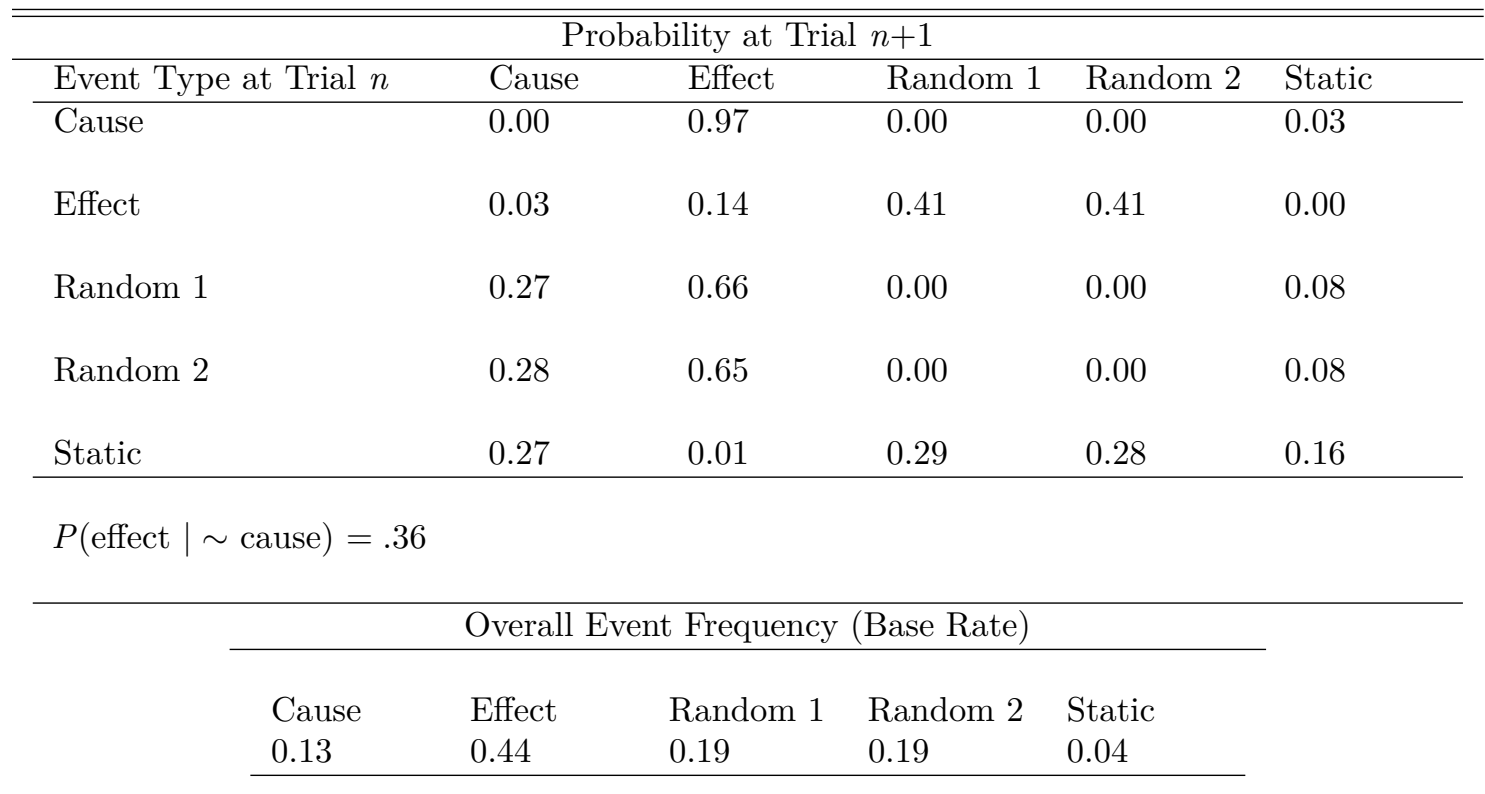


aged. Two filler questions were also presented to avoid giving the impression that the test was stuck on a single question. We chose this critical test question for several important reasons. First, by comparing order reversals, individual event frequencies in the two response options were matched. Second, in both sequence conditions, the cause-effect pair was the correct option relative to the effect-cause pair under all statistical measures: conditional probability, chunk frequency, and $\Delta P$. Between conditions, the difficulty of that choice was matched in terms of chunk frequency (63 vs. 5 / 62 vs. 6; again, we address possible effects of these minor numerical differences in Results). In terms of conditional probability, the difference between choice options was slightly larger in the low $\Delta P$ condition, which should make this choice easier, counter to our hypothesis. In terms of $\Delta P$, of course, the choice was easier in the high $\Delta P$ condition. The $\Delta P$ of the effect-cause pair was -.11 in the low $\Delta P$ condition and -.04 in the high $\Delta P$ condition.

Freeform response. To probe explicit (verbalizable) access to the sequence statistics, participants were given a freeform question asking "Did certain events follow each other more often than others? Describe any you noticed for the first set and for the second set of videos". They were also asked, "How confident are you that you detected any systematic order to the events in the [first/second] set of videos?", for each set, and responded on a $1-5$ rating scale, with $1=$ Definitely False, $3=$ Unsure, and $5=$ Definitely True.

Statistical reporting. All statistical tests reported are two-tailed, and use an alpha level of .05 for significance. Cohen's $d$ is used as a measure of effect size for the t-test, and is computed according to formulas reported in Hentschke \& Stüttgen (2011). All analysis code is available at https://osf.io/up8qz/.

\subsection{Results}

Participants performed well on the cover task in all measures of performance. Across conditions, they exhibited statistically indistinguishable numbers of hits (correct identification of rare events; high $\triangle P: M=56.20, S E=$ 2.61; low $\Delta P: M=56.24, S E=2.43)$ and false alarms (incorrect identification of common events; high $\Delta P$ : $M=6.38, S E=0.94 ;$ low $\Delta P: M=5.09, S E=0.71)$, all $p>.10$.

On the critical questions of the forced choice test (cause-effect vs. effect-cause questions), participants were above chance in the high $\Delta P$ condition $(M=$ $61.83 \%, S E=3.90 \%, t(79)=2.79, p=.007, d=0.31)$ but below chance in the low $\Delta P$ condition $(M=$ $41.67 \%, S E=3.89 \%, t(79)=-2.16, p=.034, d=$ $-0.24)$, which were significantly different from each other $(C I[8.43,29.90], t(79)=3.55, p<.001, d=0.55)$, as shown in Figure 2. Because the data are limited in range, this condition comparison was confirmed with a permutation test, which yielded $p=.004$. This supports the idea that participants had a weaker representation of the cause-effect relationship in the low $\Delta P$ condition. They were systematically incorrect on forced-choice tests for this condition, despite the fact that in both conditions, cause-effect transitions occurred twelve times as often as effect-cause transitions. There was no effect of training order (which sequence participants saw first), $p=.273$ (permutation test $p=.129$ ).

To rule out that our effects were due to the minor difference in the exact number of times participants saw a cause-effect chunk relative to an effect-cause chunk in the two conditions, we computed the difference in chunk frequency between the two conditions for each participant $(M=2)$. This difference was uncorrelated with the difference in accuracy between the two conditions $(r(78)=-0.03, p=.791)$. These minor differences thus cannot explain the effect.

As a convergent measure, we rated participants' freeform responses for whether they described the causeeffect regularity. We found that 20/80 participants described it for one sequence, but only $2 / 80$ did so for both. In fact, participants were significantly more likely to describe it for the high $\Delta P$ events $(19 / 80)$ than the low $\Delta P$ events $\left(5 / 80 ; \chi^{2}(1)=9.61, p=.002\right)$. Thus, the high $\Delta P$ condition enabled participants to better notice the predictive pattern. We observed few reports of effect-cause patterns in both conditions: there were no cases of this for the low $\Delta P$ condition, and one in the high $\Delta P$ condition. This argues against the idea that they strongly believed in the effect-cause relation in the low $\Delta P$ condition, despite the below-chance responding on the forced-choice test. Thus, although the below-chance responses in the forced-choice test are difficult to explain, this convergent measure confirms that the difference between conditions was not an anomalous artifact of the forced choice test.

Finally, participants' confidence that they noticed any systematic order among the events was not reliably above 'unsure' for either condition (high: $M=$ $3.20, S E=0.13, t(79)=1.57, p=.121$; low: $M=$ $3.06, S E=0.13, t(79)<1)$, with no effect of condition $(t(79)=1.52, p=.132)$. It is possible that participants did not recall exactly which cause-effect pattern appeared first vs. second, or thought that despite noticing one regularity, the overall order still felt random.

\subsection{Discussion}

During a VSL task, participants' learning of a highly likely transition between a cause and an effect event was weaker when the effect was also preceded by other events on other occasions (low $\Delta P$ condition), relative to when the effect was uniquely predicted by the cause (high $\Delta P$ condition). This was despite the fact that in both condi- 


\section{Experiment 1}

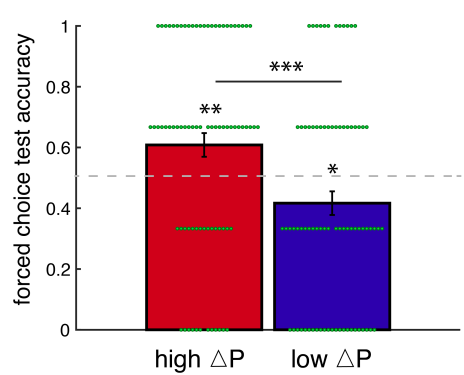

Experiment 2

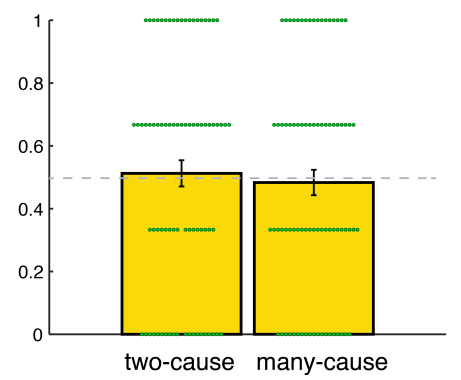

Experiment 3

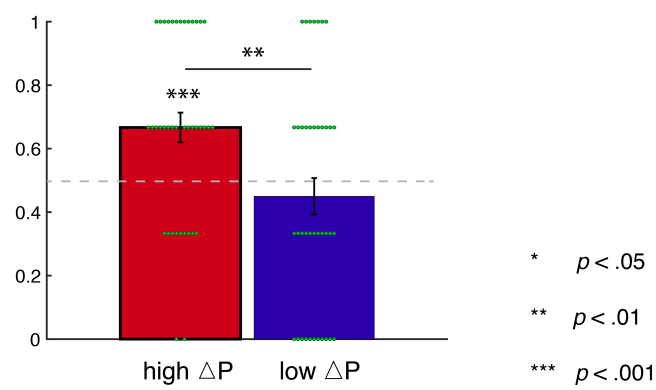

Figure 2. For each of the three experiments, we plot forced-choice test accuracy on critical questions in each pair of conditions. Green dots depict individual data-points. Dotted line is chance performance.

tions, a cause-effect transition occurred an equally high number of times. This suggests that information about additional predictors of the effect decreased participants' representation of the cause-effect predictive strength. In this way, it abstractly resembles the phenomenon of blocking seen in other learning paradigms (Kamin, 1968), and implies participants represented their learning in terms of $\Delta P$, rather than conditional or joint probability. The finding that this occurs in a VSL task is important, because it demonstrates that computations of $\Delta P$ can be an automatic and non-strategic part of learning. Answers based on chunk frequency or conditional probability were both valid, and computationally simpler, but these quantities appeared not to be available to learners to the same degree as $\Delta P$ : if they had been, responses could still have been accurate in the low $\Delta P$ condition, and should not have differed from the high $\Delta P$ condition.

We also found that unique predictive relations were more likely to be noticed, indicating that the effect of uniqueness was not operating at the level of explicit deliberation, but affected learning itself. Overall, we suggest that the computation of $\Delta P$ was a natural product of participants' incidental learning process.

These results are in line with a related finding that participants show poor memory - not just low causal ratings - for non-unique (blocked) cause-effect relations in causal reasoning tasks (Mitchell et al., 2006). They are also consistent with demonstrations of more traditional blocking effects in infants in a paradigm somewhere in between classical conditioning and statistical learning (Sobel \& Kirkham, 2006, 2007). Here we show that effects of uniqueness hold in a traditional statistical learning paradigm, characterized by sequentially presented events, incidental learning, absence of incentive or reward, and conditions which exceed the capacity to track event statistics explicitly.

A puzzling fact of these data is the below-chance performance in the low $\Delta P$ condition: participants selected the effect-cause transition more often than cause-effect. It did not appear that participants strongly believed in this effect-cause transition, as they did not mention it even once in their freeform responses. It is possible that participants may have had an inhibited representation of the cause-effect relation, or that there was an unknown pragmatic factor affecting responses. We address some of these concerns in Experiment 3, but are reassured by the convergent effects in the freeform task.

An open question is the source of this blocking-like effect. As the term 'blocking' implies, effects in traditional paradigms with two cues and one outcome are interpreted as an inference regarding which cue is responsible: the strong weight of one cue prevents the formation of another. "If Cue 1 is responsible for the outcome, then Cue 2 probably is not" (Kruschke, 2008, $p 216)$; or that "the outcome has been selectively attributed to the pretrained stimulus" (Shanks, 1985, p3). While these are meant as more or less informal descriptions of the phenomenon, there seems to be an important, though nuanced, difference between a competing cue taking responsibility for an outcome, vs. predictive weights being scaled by the outcome's base-rate. In the latter, the outcome simply happens often, rather than being already-explained by or attributed to any particular cue. We adjudicate between these possibilities in Experiments $2 \& 3$

\section{EXPERIMENT 2}

In Experiment 1's low $\Delta P$ condition, random events 1 and 2 each had a relatively strong transition probability to the effect (Figure1B; $\sim 65 \%$ ). Following the traditional interpretation of blocking, which we term the competing predictor account, participants had a uniqueness assumption about predictors, but had several options to choose from. They thus might have settled on an implicit 'explanation' that one of these events was the predictor, but this was not always the 'cause' event. Al- 
Table 2 Transition probabilities between all events and overall frequencies of each event in the two sequence conditions in Experiment 2, Two-Cause and Many-Cause. In the transition matrices, rows show the event type at trial n; columns show the probability of showing each event type at trial $n+1$. All values shown describe the actual materials shown to participants and closely resemble the idealized values used to construct the materials.

$$
\text { Many-Cause Condition }(\Delta P=.79 \%)
$$

\begin{tabular}{|c|c|c|c|c|c|c|}
\hline \multicolumn{7}{|c|}{ Probability at Trial $n+1$} \\
\hline Event Type at Trial $n$ & Cause & Effect & $\begin{array}{l}\text { Random } \\
1\end{array}$ & $\begin{array}{l}\text { Random } \\
2\end{array}$ & $\begin{array}{l}\text { Random } \\
3\end{array}$ & Static \\
\hline Cause & 0.00 & 0.96 & 0.01 & 0.01 & 0.01 & 0.01 \\
\hline Effect & 0.05 & 0.17 & 0.18 & 0.19 & 0.18 & 0.22 \\
\hline Random 1 & 0.17 & 0.19 & 0.16 & 0.17 & 0.17 & 0.14 \\
\hline Random 2 & 0.17 & 0.20 & 0.16 & 0.15 & 0.18 & 0.16 \\
\hline Random 3 & 0.18 & 0.20 & 0.16 & 0.16 & 0.15 & 0.15 \\
\hline Static & 0.25 & 0.01 & 0.19 & 0.19 & 0.18 & 0.18 \\
\hline \multicolumn{7}{|c|}{ Overall Event Frequency (Base Rate) } \\
\hline $\begin{array}{l}\text { Cause } \\
0.14\end{array}$ & $\begin{array}{l}\text { Effect } \\
0.28\end{array}$ & $\begin{array}{l}\text { Random } 1 \\
0.15\end{array}$ & $\begin{array}{l}\text { Random } 2 \\
0.15\end{array}$ & $\begin{array}{l}\text { Random } 3 \\
0.15\end{array}$ & $\begin{array}{l}\text { Static } \\
0.15\end{array}$ & \\
\hline
\end{tabular}

Two-Cause Condition $(\Delta P=.79 \%)$

\begin{tabular}{lllllll}
\hline \hline & \multicolumn{7}{c}{ Probability at Trial $n+1$} & \\
\hline Event Type at Trial $n$ & Cause & Effect & $\begin{array}{l}\text { Random } \\
1\end{array}$ & $\begin{array}{l}\text { Random } \\
2\end{array}$ & $\begin{array}{l}\text { Random } \\
3\end{array}$ & Static \\
\hline Cause & 0.00 & 0.97 & 0.01 & 0.01 & 0.01 & 0.01 \\
Effect & 0.05 & 0.01 & 0.24 & 0.24 & 0.23 & 0.22 \\
Random 1 & 0.01 & 0.95 & 0.01 & 0.01 & 0.01 & 0.01 \\
Random 2 & 0.23 & 0.01 & 0.19 & 0.17 & 0.20 & 0.21 \\
Random 3 & 0.24 & 0.01 & 0.19 & 0.19 & 0.17 & 0.20 \\
Static & 0.26 & 0.01 & 0.18 & 0.19 & 0.19 & 0.17 \\
& & & & & &
\end{tabular}

Overall Event Frequency (Base Rate)

\begin{tabular}{llllll}
\hline & & & & & \\
Cause & Effect & Random 1 & Random 2 & Random 3 & Static \\
0.14 & 0.28 & 0.15 & 0.15 & 0.15 & 0.15 \\
\hline
\end{tabular}


ternatively, the base-rate normalization account is that the cause-effect transition probability was competing not with other strong transition probabilities, but with the base-rate of the effect following any other predictor, as captured in the second term of the $\Delta P$ equation. None of the other events need to have a strong transition probability to lower the strength of the cause. Adjudicating between the competing-predictor and base-rate normalization accounts will help describe the nature of this learning process, even if its outcome is in both cases $\Delta P$.

Here, as in Experiment 1, participants were exposed to two sequences during a VSL task, each containing a high transition probability between a cause and effect ( $\sim 97 \%$; Table 2$)$. In both, the $\Delta P$ between cause and effect was of medium strength $(\sim .79)$, but for two different reasons. In the two-cause stream, the cause had one, strong competitor: another event with a high transition probability to the effect (95\%). In the manycause stream, the effect followed any of three other events, but with a fairly low probability (22\%). Thus, the cause-effect pair in both conditions had an equal $\Delta P$ value $(\sim .79)$, lower than that in the high $\Delta P$ condition of Experiment 1, but two different sources of competition: a strong alternative or a high overall base-rate.

Any differences between conditions would indicate that one kind of competition was more effective than another, keeping $\Delta P$ equal. If uniqueness effects are due to a salient alternative predictor, participants should perform worse in the two-cause stream relative to the many-cause stream. On the other hand, if uniqueness effects are driven by base-rate normalization, learning should be relatively poor in both conditions, since a single strong predictor and multiple weak predictors both raise the overall base-rate.

\subsection{Methods}

\subsubsection{Participants}

107 participants were recruited and tested via Amazon Mechanical Turk. Participants provided electronic consent and procedures were approved by the Institutional Review Board of the University of Pennsylvania. Compensation was $\$ 3$, with a bonus of up to $\$ 2.50$ based on task accuracy. Participants were excluded if they had previously participated in a related experiment or this one (7), for failing an attention measure (19), or for missing data (1). Data from the remaining 80 participants are reported here (47 female, age $M=34$, range $19-71)$.

\subsection{Stimuli \& Procedure}

Stimuli and procedures were similar to Experiment 1, except that each sequence contained 5 event types rather than 4 , and the sequence was slightly shorter (450 events vs. 500). The transition matrices are shown in Table 2 ; the main difference is that the two-cause condition had a $95 \%$ transition probability between random event 1 and the effect; while the many-cause condition had a $\sim 22 \%$ transition probability between any of three random events and the effect. Conditions had similar chunk frequencies for cause-effect and effect-cause pairs, and similar $\Delta P$ value between cause and effect $(\sim .79)$.

\subsection{Results}

Participants performed well on the cover task for both sequences. Although they exhibited statistically indistinguishable numbers of false alarms on common events for both conditions (two-cause: $M=5.35, S E=$ 0.78 ; many-cause : $M=4.33, S E=0.73, p>.2)$, they did show significantly more hits on rare events in the many-cause condition (two-cause: $M=51.20, S E=$ 2.58 ; many-cause: $M=56.90, S E=2.45, t(79)=$ $-2.94, p=0.004)$. Overall task accuracy did not differ reliably $(t<1)$.

On the critical trials in the forced-choice test, performance was not reliably different from chance in either condition (two-cause: $M=51.25 \%, S E=4.17, t(79)<$ 1; many-cause: $M=48.33 \%, S E=4.04, t(79)<1)$ with no significant difference between them $(t<1)$, as shown in Figure 2. This result was confirmed with a permutation test, yielding $p=.29$. Thus, in both conditions, learning was not reliably observed, although the null result should be interpreted with caution.

Confidence ratings did not statistically differ from unsure (two-cause: $M=2.80, S E=0.13, p>.10$; manycause: $M=2.80, S E=0.13, p>.10)$ or from each other $(t<1)$. The same number of participants noticed the cause-effect pattern in the two-cause condition $(M=9)$ as in the many-cause condition $(M=9)$.

\subsection{Discussion}

When shown a cause-effect relation accompanied by either an alternative strong predictor of the same effect (two-cause condition) or several weak predictors (manycause condition), participants showed equally poor learning of the effect-cause relation in both. It seemed not to matter for learning whether there was a salient alternative cause, or simply a higher base rate (overall high frequency of occurrence) of the effect. Although this was a null result, this pattern of data is more consistent with a base-rate normalization account than a competing-predictor account, the latter of which would predict that the two-cause condition should have lessened belief in the cause-effect relationship. Nonetheless, this conclusion requires a more direct test, as this null finding could simply be due to participants not learning for other reasons. Experiment 3 therefore compares a high and low $\Delta P$ many-cause condition to show that a 
single strong alternative predictor is not necessary to see this effect.

\section{EXPERIMENT 3}

We aimed to demonstrate that multiple, weak predictors that raise the overall base-rate of the effect significantly decrease learning in VSL relative to a high $\Delta P$ condition similar to Experiment 1 . We created a many-cause low $\Delta P$ condition in which 3 events preceded the effect, but all with a transition probability lower than the effect's base-rate (Table 3). We compared it to a high $\Delta P$ condition similar to that in Experiment 1. We also improved the design in a few ways, described in Methods. Most importantly, we ran the conditions in a betweensubject design, as we suspected that the below-chance response in the low $\Delta P$ condition of Experiment 1 might have been due to participants being exposed to both conditions. Piloting with these materials in a withinsubject design did show below-chance accuracy in the low $\Delta P$ condition (as well as a difference between conditions, as predicted). We wanted to ensure that we could see a significant effect of condition without significant belowchance performance in low $\Delta P$, so we report this version here.

\subsection{Methods}

\subsubsection{Participants}

118 participants were recruited and tested via Amazon Mechanical Turk. Participants provided electronic consent and procedures were approved by the Institutional Review Board of the University of Pennsylvania. Compensation was $\$ 2.50$, with a bonus of up to $\$ 2.50$ based on task accuracy. Participants were excluded if they had previously participated in a related experiment or this one (11), for failing an attention measure (23), or for missing data (4). Data from the remaining 80 participants are reported here (40 female, age $M=35$, range $19-61)$.

\subsubsection{Stimuli \& Procedure}

Stimuli and procedures were largely similar to Experiments $1 \& 2$, with a few differences. Participants were randomly assigned to either the high $\Delta P$ condition or the low $\Delta P$ condition. We increased the preview length to 150 events, to mitigate a dip in cover task accuracy when showing 5 vs 4 different event types, and increased the overall sequence length to 600 , to further mitigate below-chance accuracy. Table 3 shows the transition matrices used in the two conditions and their associated $\Delta P$ values. The number of cause-effect chunks was similar (high $\Delta P: M=97.65$; low $\Delta P: M=98.25$ ), as was their conditional probability (Table 3 ), and there were 0 effect-cause chunks. Thus, the difference between these was also similar.

\subsubsection{Results 8 Discussion}

Participants were highly accurate on the cover task, with no difference between conditions in terms of hits on rare events (high $\triangle P: M=51.39, S E=3.63$; low $\Delta P: M=47.31, S E=4.07 ; t<1$ ) or false alarms on common events (high $\Delta P: M=6.51, S E=1.89$; low $\Delta P: M=5.96, S E=1.23 ; t<1)$.

On critical trials of the forced-choice test, participants were significantly above-chance in the high $\Delta P$ condition $(M=66.67, S E=4.68, C I[0.5732,0.7602], t(39)=$ $3.61, p<.001, d=0.57)$ but not above or below chance in the low $\Delta P$ condition $(M=45.00, S E=$ $5.74, C I[0.3354,0.5646], t(39)=-0.88, p=.383, d=$ $-0.14)$, with a significant difference between them, $t(78)=2.96, p=.004, d=0.66$. This was confirmed with a permutation test, yielding $p=.004$. Results are shown in Figure 2. We thus suspect that the belowchance accuracy in the low $\Delta P$ condition seen previously was driven by some feature of a within-subjects design.

Confidence ratings were not above 'unsure' for either condition (high $\Delta P: M=3.00$; low $\Delta P: M=3.04$, $t$ 's $<1$ ) and did not differ from each other, $t<1$. In freeform responses, noticing was relatively rare $(12 / 80$ participants), with no difference between conditions, $\chi^{2}=0.392, p>.50$. It is likely that, given the high individual variability in noticing, and the low rate overall, a between-subjects design was too under-powered to show an effect. It is also very likely that the increased number of events - 5 rather than 4 - decreased the overall rate at which participants managed to notice any regularities.

In summary, we were able to successfully replicate the forced-choice results from Experiment 1, without significantly below-chance accuracy in the low $\Delta P$ condition. This suggests that the effect of $\Delta P$ is over and above any additional factors leading to below-chance responding. We also improved the design with more precisely matched materials in minor statistical details. More importantly, we found these robust effects of $\Delta P$ even when the low $\Delta P$ between cause and effect was due not to any strong individual predictors, but simply to the increased base-rate of the effect, confirming the results of Experiment 2 and supporting the base-rate normalization model.

\section{COMPUTATIONAL MODELING}

The $\Delta P$ equation describes the outcome of learning, but not the process by which learners arrive at it (Shanks, 1995). The Rescorla-Wagner (R-W) model is a highly influential model of this process (Rescorla \& Wagner, 1972). However, it explains traditional blocking effects only in some situations (Kruschke, 2008). Here we adapted the R-W model to handle sequential stimuli to test whether it could account for the effects we see in VSL, and if not, what minimal change could account for them. Indeed, we found that a sequential adapta- 
Table 3 Transition probabilities between all events and overall frequencies of each event in the two sequence conditions in Experiment 3, High $\Delta P$ and Many-Cause Low $\Delta P$. In the transition matrices, rows show the event type at trial n; columns show the probability of showing each event type at trial $n+1$. All values shown describe the actual materials shown to participants and closely resemble the idealized values used to construct the materials.

Many-Cause Low $\Delta P$ Condition $(\Delta P=.72 \%)$

\begin{tabular}{lllllll}
\hline \hline & \multicolumn{7}{c}{ Probability at Trial $n+1$} & \\
\hline Event Type at Trial $n$ & Cause & Effect & $\begin{array}{l}\text { Random } \\
1\end{array}$ & $\begin{array}{l}\text { Random } \\
2\end{array}$ & $\begin{array}{l}\text { Random } \\
3\end{array}$ & Static \\
\hline Cause & 0.00 & 0.97 & 0.01 & 0.01 & 0.01 & 0.00 \\
Effect & 0.00 & 0.21 & 0.27 & 0.27 & 0.26 & 0.00 \\
Random 1 & 0.37 & 0.35 & 0.05 & 0.04 & 0.05 & 0.14 \\
Random 2 & 0.37 & 0.35 & 0.04 & 0.04 & 0.04 & 0.16 \\
Random 3 & 0.42 & 0.33 & 0.03 & 0.03 & 0.04 & 0.15 \\
Static & 0.29 & 0.00 & 0.19 & 0.18 & 0.17 & 0.17 \\
\end{tabular}

Overall Event Frequency (Base Rate)

\begin{tabular}{llllll} 
Cause & Effect & Random 1 & Random 2 & Random 3 & Static \\
0.17 & 0.16 & 0.19 & 0.21 & 0.19 & 0.08 \\
\hline
\end{tabular}

High $\Delta P$ Condition $(\Delta P=.98 \%)$

\begin{tabular}{lllllll}
\hline \hline & \multicolumn{7}{c}{ Probability at Trial $n+1$} & \\
\hline Event Type at Trial $n$ & Cause & Effect & $\begin{array}{l}\text { Random } \\
1\end{array}$ & $\begin{array}{l}\text { Random } \\
2\end{array}$ & $\begin{array}{l}\text { Random } \\
3\end{array}$ & Static \\
\hline Cause & 0.00 & 0.98 & 0.01 & 0.01 & 0.01 & 0.00 \\
Effect & 0.00 & 0.01 & 0.32 & 0.35 & 0.32 & 0.00 \\
Random 1 & 0.26 & 0.00 & 0.20 & 0.23 & 0.21 & 0.11 \\
Random 2 & 0.25 & 0.00 & 0.22 & 0.21 & 0.21 & 0.11 \\
Random 3 & 0.24 & 0.00 & 0.21 & 0.23 & 0.23 & 0.10 \\
Static & 0.28 & 0.00 & 0.17 & 0.20 & 0.20 & 0.16 \\
& & & & & &
\end{tabular}

Overall Event Frequency (Base Rate)

\begin{tabular}{llllll}
\hline & & & & & \\
Cause & Effect & Random 1 & Random 2 & Random 3 & Static \\
0.17 & 0.37 & 0.13 & 0.13 & 0.13 & 0.08 \\
\hline
\end{tabular}


With Normalization

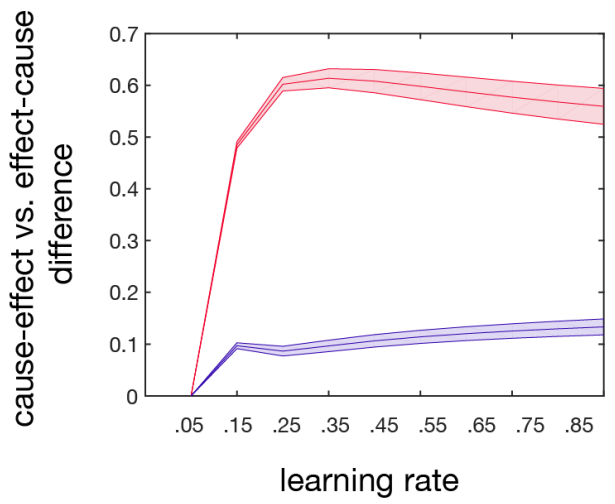

Without Normalization

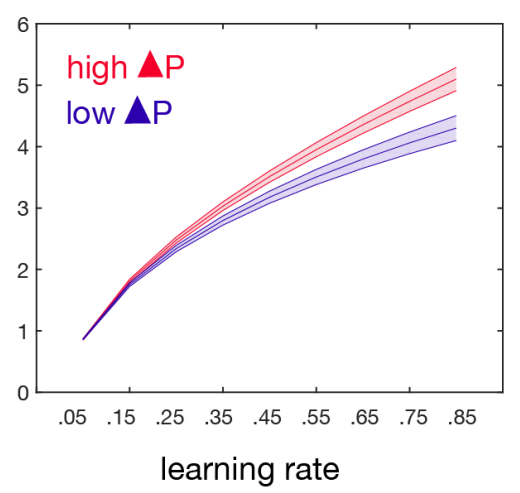

Figure 3. Learning shown in our adapted R-W model, with normalization (left) and without (right). Learning is taken as the difference in weights between likely (cause-effect) pairs relative to unlikely (effect-cause) pairs. This difference is attenuated by non-uniquess in the low $\Delta P$ condition (blue line) vs. high $\Delta P$ condition (red line), but only in the model with normalization.

tion of the standard R-W model did not account for our effect of condition in Experiment 1. However, we found that a very simple normalization step circumvents this problem, and that this solution is consistent with a straightforward cognitive account of how we compute $\Delta P$, namely, normalization among predictor weights.

\subsection{Model Details}

We assume that learners acquire associative weights among all pairs of stimuli and use differences in pairwise associative weights to solve the forced choice task. As such, the model learns the weight of links among all pairs of stimuli 1 to $s$, represented by matrix $\mathbf{W}$ with $s \times s$ entries. We allow the learner to know the number of stimuli and to begin with the assumption that all transitions are equally likely; thus, all entries in $\mathbf{W}$ are initially set to $1 / s$. Learning operates sequentially over observed input stream o, which consists of a sequence of the stimuli from set $\{1: s\}$, as shown to human learners. At each observation $\mathbf{o}_{i}$, stimulus $i$ is shown. The model compares this observation with its prediction for $\mathbf{o}_{i}$ on the basis of the preceding $n$ stimuli $\left(\mathbf{o}_{i-n} \ldots \mathbf{o}_{i-1}\right)$, which consists of stimuli $k_{1: n}$ also in set $\{1: s\}$ and the weights between $k_{1: n}$ and $i$, as represented in $\mathbf{W}$. Its error in anticipating the stimulus is used to adjust the entries in $\mathbf{W}$ between stimuli $k_{1: n}$ and stimulus $i$, which are then used in subsequent predictions. Formally, the degree of anticipation of stimulus $i$ is given by:

$$
a_{i}=\Sigma_{k=1}^{n} \mathbf{w}_{k i}
$$

For simplification, we set $n=1$, such that we simply use the entry between the current stimulus $i$ and the single preceding stimulus $k$. This does not affect results, since our sequences have the Markov property. The error is computed by:

$$
d_{i}=1-a_{i}
$$

Because stimuli occur in binary fashion, their observed value is always 1 . If this was well anticipated by the previous stimuli, $\mathrm{a}_{i}$ should be close to 1 , resulting in a low error. The corresponding entries in $\mathbf{W}$ are adjusted by this error multiplied by a learning rate parameter $\alpha$ :

$$
\Delta \mathbf{W}_{k i}=\alpha d_{i}
$$

This concludes the steps of the basic model. For the normalized model, we additionally normalized the columns of $\mathbf{W}$ (input weights to each event) such that they sum to 1 .

\subsection{Results \& Discussion}

The event sequences given to human participants in Experiment 1 served as inputs to the model. The outputs were the learned matrices $\mathbf{W}$, for each set of materials. We assumed that forced-choice behavior reflects the relative weight of links between cause-effect and effectcause pairs, and thus, subtracted these entries in $\mathbf{W}$. The difference between them was taken to reflect learning, and we compared learning in the high and low $\Delta P$ conditions.

The standard model did not show a robust effect of condition: it showed small though significant effects only at high learning rates $(>0.35)$, but not at the others (Figure 3). We thus created a normalized model, where a step at each trial required the set of weights to each event from all others to sum to 1 . This meant that weights could trade off, such that stronger AX weights would naturally reduce BX weights. We found that this simple adaptation enabled us to capture the difference between conditions: we found significantly 
stronger weights for cause-effect than effect-cause links at all learning rates $(.05-.85$; Figure 3$)$. For example, at learning rate $\alpha=0.5$, we saw a significantly better learning in the high $\Delta P$ condition (cause-effect $M=0.70, S E=0.02$; effect-cause $M=0.11, S E=$ $0.01, t(79)=21.92, p<.001)$ than in the low $\Delta P$ condition (cause-effect $M=0.19, S E=0.01$, effect-cause $M=0.07, S E=0.01, t(79)=9.07, p<.001,(t(79)=$ $17.35, p<.001)$. The same pattern is seen consistently across settings of the learning rate parameter. This is important because, given the relatively weak human learning, we expect that the actual learning rate is fairly low.

Similar effects arise when using materials from Experiment 3 (here shown with a learning rate of .1 , but which are consistent at other rates): the high $\Delta P$ condition shows better learning of the cause-effect vs effect-cause associative strength $(M=0.5736, S E=$ $0.01)$ than the low $\Delta P$ condition $(M=0.087, S E=$ $0.01, C I[0.4621,0.5119], t(79)=38.97, p<.001, d=$ 6.11). Regarding Experiment 2, we find that the model, unlike participants, does exhibit stronger learning in the two-cause condition $(M=0.253, S E=0.01)$ than the many-cause condition $(M=0.128, S E=$ $0.01, C I[0.1046,0.1449], t(79)=12.32, p<.001, d=$ $1.59)$, albeit with an effect size a quarter the magnitude. This suggests that such effects could potentially be harder to detect in human participants and would require twice the sample size we used here. Nonetheless, this modeling result bolsters the prediction that uniqueness effects may arise most strongly from a high base rate (many-cause condition) rather than a second, single, competing cause (two-cause condition). Such a result in human participants could bolster our argument that uniqueness effects are driven by base-rate normalization.

The finding that a standard R-W model does not account for our effects of uniqueness is consistent with prior observations that this model has difficulty accounting for certain types of blocking: specifically, those which involve updating weights for stimuli using trials in which those stimuli are not actually present-e.g., using B+ trials to update $\mathrm{A}+$ representations. This is known as retrospective re-evaluation (Kruschke, 2008; Shanks, 1985). Several adaptations of R-W have been offered for this reason (see Shanks, 2010, for a review). The most appropriate for our sequential, VSL task is the Kalman filter, a Bayesian version of R-W (Kruschke, 2008), which assumes that learners represent the relation between predictors and an outcome as a set of multivariate normal probabilities, i.e., as a mean and a variance for a set of weights. Because probabilities sum to 1 , the strengths of predictors naturally trade off. Indeed, the Kalman filter readily explains our effects (cause-effect vs. effect-cause difference, high $\Delta P$ condition, $M=0.317, S E=0.068$, low $\Delta P$ condition $M=0.000, S E=0.000, C I[0.1815,0.4540], t(79)=$ $4.64, p<.001, d=0.73)$. However, our own model pro- vided a key demonstration that the essential feature for obtaining the $\Delta P$ effect is specifically normalization among predictors, rather than the computation of uncertainty or other features of probability distributions per se.

Whether implicitly learned associative weights are represented as probabilities is a separate question; here we are concerned with finding the simplest rather than the most complete model, in order to identify what algorithm could capture uniqueness effects most straightforwardly. Learners appear to also have explicit representation of uncertainty in more deliberate learning tasks, which only the Kalman filter naturally captures because it is Bayesian (Kruschke, 2008). We plan to test whether learners in VSL tasks also represent uncertainty in future work.

\section{CONCLUSIONS}

Our key finding was that participants learned pairwise associations between events not based on their conditional probability, but rather on a more inferentially complex quantity, $\Delta P$. This reflected whether a high conditional probability was unique; that is, stronger than other predictors of the same outcome. If participants had computed conditional probabilities or chunk frequencies, they could have used these quantities to accurately solve the forced-choice test in both high and low $\Delta P$ conditions. That they did not do so suggests they came to learn that pairwise relation in terms of $\Delta P$ rather than these other statistical quantities. This result is at odds with a common assumption in the statistical learning literature (Fiser \& Aslin, 2001; Orbán et al., 2008) but in line with other literatures on learning and reasoning.

The importance of $\Delta P$ has been long reported in classical conditioning and causal reasoning paradigms by using blocking manipulations (Allan, 1980; Cheng, 1997; Shanks, 1985, 1995; Spellman, 1996), but these prior effects could be driven by how participants reason over information they already know, rather than how they learn and encode information in memory (Mitchell et al., 2009). In statistical learning paradigms, the experiential input rapidly exceeds learners' ability to track the specific sequence of what they have seen, and testing reflects what they have encoded in memory. We find that despite these differences, blocking-like effects emerge during statistical learning. This suggests that the uniqueness of a relation may be an inherently important statistical property that the mind spontaneously computes as part of how it forms relational memories. This implies a broader commonality between statistical learning and both classical conditioning and causal reasoning than perhaps previously assumed.

We show that uniqueness can be reduced by either a strong competing predictor or an overall high base rate of the effect event. Both can be seen as the result of nor- 
malization: the assumption that predictors of the same effect trade off, and to be considered effective, must raise the probability of the effect above that of the others, or, its rate of occurrence otherwise. A simple normalization step to the classic R-W learning algorithm was sufficient to account for these effects. Broadly speaking, the conversion to relative rather than absolute values is a cognitively realistic and adaptive mechanism that likely applies widely in the brain.

Overall, our findings bring learning in VSL tasks in closer contact with the rich literature in classical conditioning and causal reasoning, despite differences in the nature of these learning circumstances. These findings suggest that similar computations take place incidentally and automatically to govern learning, and thus describe how we register the naturally occurring statistics of our observed world.

\section{AUTHOR NOTE}

A previous, shorter version of this paper appeared in the Proceedings of the 40th Annual Meeting of the Cognitive Science Society and was presented as a talk at the Computational Cognitive Neuroscience Conference in Philadelphia, Sept 5-8, 2018.

\section{DATA AND CODE AVAILABILITY}

The data, stimuli, task materials, and code for all experiments, and code for the computational models, are all publicly available at the Open Science Framework repository at https://osf.io/up8qz/ with DOI 10.17605/OSF.IO/UP8QZ.

\section{ACKNOWLEDGEMENTS}

This work was supported by NIH grant R01DC015359 to S.L.T-S. We thank Michael F. Frank, Daphna Shohamy, and Jiaying Zhao for helpful discussion of these findings.

\section{REFERENCES}

Allan, L. G. (1980). A note on measurement of contingency between two binary variables in judgment tasks. Bulletin of the Psychonomic Society, 15(3), 147-149.

Brady, T. F., \& Oliva, A. (2008). Statistical learning using real-world scenes: extracting categorical regularities without conscious intent. Psychological Science, $19(7), 678-685$.

Buchsbaum, D., Griffiths, T. L., Plunkett, D., Gopnik, A., \& Baldwin, D. (2015). Inferring action structure and causal relationships in continuous sequences of human action. Cognitive Psychology, 76, 30-77.

Cheng, P. W. (1997). From covariation to causation: A causal power theory. Psychological Review, 104(2),
367-405.

Endress, A. D., \& Langus, A. (2017). Transitional probabilities count more than frequency, but might not be used for memorization. Cognitive Psychology, 92, $37-64$.

Fiser, J., \& Aslin, R. N. (2001). Unsupervised Statistical Learning of Higher-Order Spatial Structures from Visual Scenes. Psychological Science, 12(6), 499-504.

Fiser, J., \& Aslin, R. N. (2002). Statistical learning of higher-order temporal structure from visual shape sequences. Journal of Experimental Psychology: Learning, Memory, and Cognition, 28(3), 458-467.

Frank, M. C., Goldwater, S., Griffiths, T. L., \& Tenenbaum, J. B. (2010). Modeling Human Performance in Statistical Word Segmentation. Cognition, 117(2), 107-125.

Gershman, S. J. (2015). Reinforcement learning and causal models. Oxford Handbook of Causal Reasoning, 1-32.

Griffiths, T. L., \& Tenenbaum, J. B. (2005). Structure and strength in causal induction. Cognitive Psychology, 51(4), 334-384.

Kamin, L. J. (1968). "Attention-like" processes in classical conditioning. In M. Jones (Ed.), Miami symposium on the prediction of behavior: Aversive stimulation (pp. 9-31). Coral Gables, FL: University of Miami Press.

Kim, R., Seitz, A., Feenstra, H., \& Shams, L. (2009). Testing assumptions of statistical learning: Is it longterm and implicit? Neuroscience Letters, 461(2), $145-149$.

Kruschke, J. K. (2008). Bayesian approaches to associative learning: From passive to active learning. Learning and Behavior, 36(3), 210-226.

Mitchell, C. J., De Houwer, J., \& Lovibond, P. F. (2009). The propositional nature of human associative learning. Behavioral and Brain Sciences, 32(02), 183.

Mitchell, C. J., Lovibond, P. F., Minard, E., \& Lavis, Y. (2006). Forward blocking in human learning sometimes reflects the failure to encode a cue-outcome relationship. Quarterly Journal of Experimental Psychology, 59(5), 830-844.

Orbán, G., Fiser, J., Aslin, R. N., \& Lengyel, M. (2008). Bayesian learning of visual chunks by human observers. Proceedings of the National Academy of Sciences, 105(7), 2745-2750.

Rescorla, R. (1988). Pavlovian Conditioning: It's Not What You Think It Is. Americal Psychologist, 43(3), 151-160.

Rescorla, R., \& Wagner, A. (1972). A theory of Pavlovian conditioning: Variations in the effectiveness of reinforcement and nonreinforcement. Classical Conditioning II Current Research and Theory, 21(6), 64-99. 
Saffran, J. R., Aslin, R. N., \& Newport, E. L. (1996). Statistical learning by eight-month-old infants. Science, 274(5294), 1926-1928.

Shanks, D. R. (1985). Forward and backward blocking in human contingency judgement. Quarterly Journal of Experimental Psychology, 37(B), 1-21.

Shanks, D. R. (1995). The Psychology of Associative Learning. Cambridge, UK: Cambridge University Press.

Shanks, D. R. (2010). Learning: From Association to Cognition. Annual Review of Psychology, 61(1), 273-301.

Sobel, D. M., \& Kirkham, N. Z. (2006). Blickets and babies: the development of causal reasoning in toddlers and infants. Developmental Psychology, 42(6), 1103-1115.

Sobel, D. M., \& Kirkham, N. Z. (2007). Bayes nets and babies: Infants' developing statistical reasoning abilities and their representation of causal knowledge. Developmental Science, 10(3), 298-306.

Spellman, B. A. (1996). Acting as intuitive scientists: Contingency judgments are nade while controlling for alternative potential causes. Psychological Science, 7(6), 337-342.

Turk-Browne, N. B., Jungé, J., \& Scholl, B. J. (2005). The automaticity of visual statistical learning. Journal of Experimental Psychology. General, 134(4), $552-564$. 